\title{
Phylogenetic and Phylogeographic Analysis of DENV-1 Genotype I in Asian-Pacific Region
}

\author{
Deyan Yang ${ }^{1, *}$, Haiqi Wang ${ }^{1}$, and Bin Yan ${ }^{2}$ \\ ${ }^{1}$ School of Marine and Spatial information, China University of Petroleum (east China), Qingdao 266580, China \\ ${ }^{2}$ Institute of Geographic Sciences and Natural Resources Research, CAS, Beijing 100101, China
}

\begin{abstract}
Objective: To clarify the evolutionary characteristics and transmission mode of DENV-1 genotype I dengue virus in the Asia-Pacific region. Methods: 200 DENV-1 genotype I dengue viruses in the Asia-Pacific region from 2010 to 2014 and 2015 to 2019 were used for phylogenetic analysis and bayesian phylogeographic analysis using a series of molecular biology software. Results: DENV-1 genotype I dengue virus spread frequently between coastal cities in China and Southeast Asian countries. The evolution rate of DENV-1 genotype I dengue virus during 2010-2014 was $5.73 \times 10^{4}$ (/site/year,95\%CI), and the time to the most recent common ancestor was 1970-09-14.And the evolution rate of virus during 2015-2019 was $5.25 \times 10^{4}$ (/site/year,95\%CI), and the time to the most recent common ancestor was $1978-10-30$. The virus spatial diffusion pathways shows the phenomenon that the dengue virus of this genotype is widespread in the Asia-Pacific region, the spread of the dengue virus between the coastal provinces and some inland provinces of China, and Southeast Asian countries. The study results indicated that the monitoring and preventive measures should be strengthened to avoid the threat of personal safety caused by large-scale spread of DENV-1 in China.
\end{abstract}

\section{Introduction}

Dengue virus (DENV) is an arbovirus of the Flaviviridae of the Flaviviridae family. It has four serotypes with different antigens(DENV-1,DENV-2,DENV-3,DENV-4). Each serotype is further divided into 4 or 5 genotypes according to its genetic diversity. It is spread by Aedes mosquitoes. Dengue virus cause dengue fever (DF) and dengue hemorrhagic fever (DHF) with high morbidity and mortality [1]. With the global warming and the rapid development of tourism, the geographical distribution of the virus vector Aedes is also expanding. The infection of DENV is spreading rapidly around the world, and more than half of the world's population is at risk of infection. It has become one of the most harmful media diseases in the world [2]. One-third of the world's people live in dengue risk areas [3]. Dengue fever is currently prevalent in more than 100 tropical and subtropical countries and regions between $30^{\circ}$ north and south latitudes. Africa, the United States, the central Mediterranean, Southeast Asia and the Western Pacific are high-risk areas. About 1.8 billion (over 70\%) of high-risk populations of dengue fever live in Southeast Asia and the Western Pacific, accounting for nearly 75\% of the current global dengue fever [4,5].

Dengue virus E protein is the main envelope protein, covering the surface of the virion. It determines the tissue affinity of the virion and directly affects the combination of the virus and the host cell. Moreover, the E protein determines the transmission ability of the virus, and is an important component that causes a protective immune response and immune pathological damage. Therefore, the construction of the dengue virus $\mathrm{E}$ gene phylogenetic tree is of great significance for analyzing the diversity of genes and exploring the temporal and spatial transmission of the virus[6].

\section{Materials and methods}

\subsection{Virus sequence acquisition}

First, the DENV-1 sequence data in the Asia-Pacific region was collected from the GenBank database. Preliminary processing with $\mathrm{R}$ was used to obtain the $\mathrm{E}$ gene sequence data of DENV-1 genotype I virus from 21 countries or regions in the Asia-Pacific region with a total of 3,078. Then the data was further screened according to the two time periods of 2010-2014 and 2015-2019, and a total of 200 gene sequences were obtained after excluding sequences with a similarity of $99.99 \%$. Countries or regions with insufficient sample size are eliminated.It contains information such as the time and location of the isolation of the virus isolate. The location and quantity of gene sequence data are shown in Table 1 and 2.

Table.1 Statistics of E gene sequence from 2010 to 2014.

\begin{tabular}{cccc}
\hline China & $\begin{array}{c}\text { Sequence } \\
\text { number }\end{array}$ & $\begin{array}{c}\text { Other } \\
\text { countries }\end{array}$ & $\begin{array}{c}\text { Sequence } \\
\text { number }\end{array}$ \\
\hline
\end{tabular}

\footnotetext{
* Corresponding author: wanghaiqi@upc.edu.cn
} 


\begin{tabular}{cccc}
\hline Guangdong & 9 & Australia & 7 \\
Guangxi & 4 & Indonesia & 25 \\
Zhejiang & 2 & Thailand & 11 \\
Yunnan & 11 & Japan & 1 \\
Fujian & 1 & Singapore & 8 \\
& & Sri Lanka & 2 \\
& Malaysia & 1 \\
& Vietnam & 9 \\
& New & 7 \\
& Caledonia & 2 \\
\hline
\end{tabular}

Table.2 Statistics of E gene sequence from 2015 to 2019.

\begin{tabular}{cccc}
\hline China & $\begin{array}{c}\text { Sequence } \\
\text { number }\end{array}$ & $\begin{array}{c}\text { Other } \\
\text { countries }\end{array}$ & $\begin{array}{c}\text { Sequence } \\
\text { number }\end{array}$ \\
\hline Guangdong & 20 & Australia & 1 \\
Zhejiang & 11 & Indonesia & 3 \\
Henan & 15 & Thailand & 12 \\
Jiangxi & 2 & Singapore & 10 \\
& & Vietnam & 8 \\
& New & 1 \\
& Caledonia & 11 \\
& India & 1 \\
& & Myanmar & 3 \\
\hline
\end{tabular}

\subsection{Phylogenetic analysis}

Bayesian Markov chain Monte Carlo (MCMC) method was used to analyze the DENV-1 genotype I sequence with the BEAST software package v 1.10 [7] .First, each viral gene sequence was renamed so that the sequence name contains sequence ID, genotype, spatiotemporal information. Then the G-INS-i strategy was selected in MAFFT_v7.471 software [8] to perform dengue virus multiple sequence alignment. The aligned dengue virus sequence data was imported into the nucleotide substitution model selection software modelgenerator v851, the best-fit nucleotide substitution model for the data was selected based on the AIC standard, and the nucleotide substitution model combination was finally determined: GTR+Gamma+ Invariant.Based on MCMC method, the unrelaxed lognormal molecular clock and the GTR $+\mathrm{G}+\mathrm{I}$ substitution model are selected to infer the pedigree geographic structure and spatiotemporal dynamics of this genotype DENV-1 virus. On the regional scale, the Bayesian MCMC was iterated 100,000,000 generations, the sampling frequency was set to be 10,000 , and totally 10,000 samples were generated. Then convergence of parameters of the results was identified in the Tracer_v1.7.1 software, and ensure that the effective sample size (ESS) exceeding 200. The trees are merged in TreeAnnotator_v1.10.4. And the burn-in parameter was set to be $25 \%$. The editing and beautification of MCC tree is carried out with the FigTree_v1.4.4.

\subsection{Phylogeographic analysis}

In order to construct the temporal and spatial dynamics and transmission of dengue virus, the bayesian stochastic search variable selection (BSSVS) was used to provide evidence for statistically supported virus diffusion [9]. The geographic coordinate file of the virus sequence and the log file of the BEAST are imported to calculate the migration path, rate and $\mathrm{BF}$ value between different regions in spreaD3_v0.9.7.1.

Four levels of support for the virus transmission path are divided according to the combination of both $\mathrm{BF}>3$ and the indicator $>0.5$ to determine whether there is a statistically significant pathogen migration association between the two locations. $\mathrm{BF}>1000$ indicates decisive support (highest), $100<\mathrm{BF}<1000$ indicates exceedingly strong support (higher), $10<\mathrm{BF}<100$ indicates strong support (normal), $3<\mathrm{BF}<10$ indicates support (low). The size of the $\mathrm{BF}$ value represents the support rate of the virus migration pathways [10]. After statistics of the main virus migration paths, Adobe Illustrator 2020 was used to draw the dynamic map of the virus's temporal and spatial difussion.

\section{Results}

\subsection{Bayesian phylogenetic and Evolutionary characteristics analysis}

The results of the MCC tree constructed by a total of 200 DENV-1 genotype I virus E gene sequences from 20102014 and 2015-2019 are shown in Figure 1.

The evelutionary rate (The number of variations per nucleotide base position per year) of DENV-1 genotype I dengue virus during 2010-2014 was $5.73 \times 104$ (/site/year,95\%CI) , and the time to the most recent common ancestor was 1970-09-14.And the evolution rate of DENV-1 genotype I dengue virus during 20152019 was $5.25 \times 104$ (/site/year,95\%CI), and the time to the most recent common ancestor was 1978-10-30.

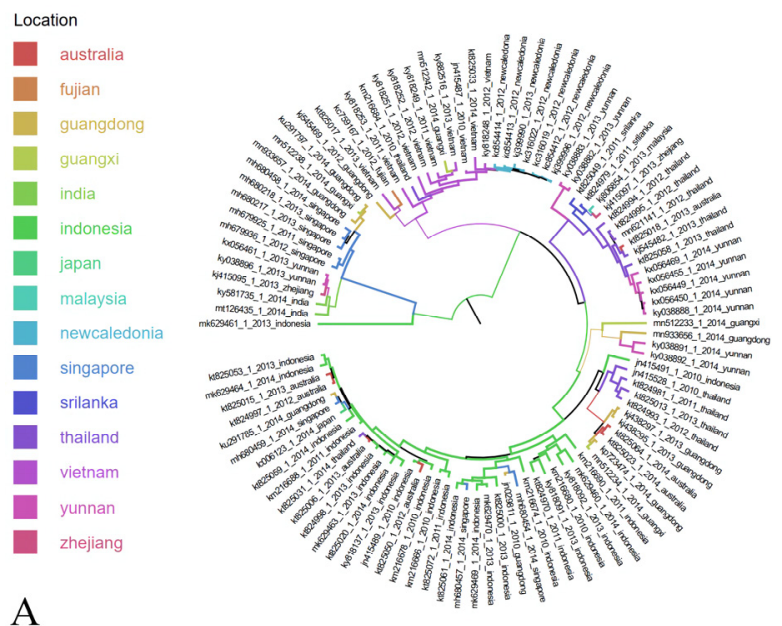




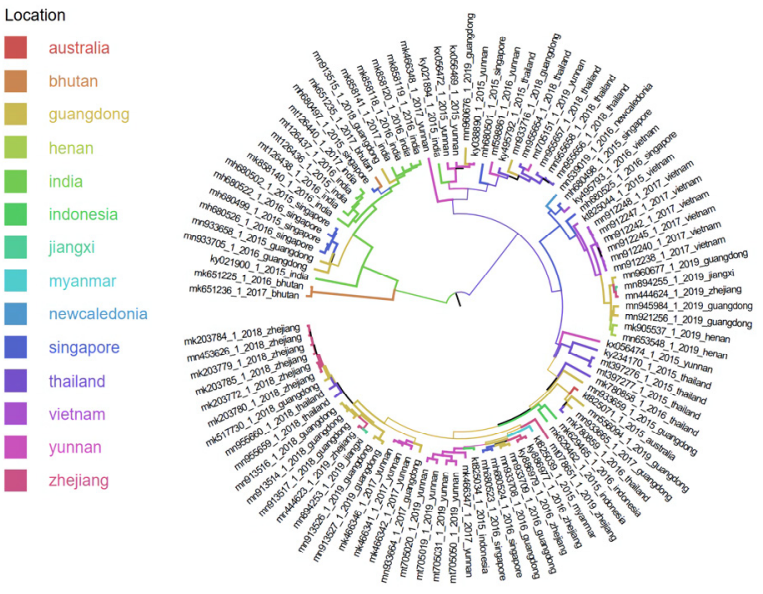

B

Fig.1 Maximum clade credibility (MCC)trees summarized for DENV-1 genotype I virus dataset. A, B represented the MCC trees of 2010-2014, 2015-2019, respectively. The colors of the branches corresponded to their probable geographic location.

\subsection{Discrete phylogeography analysis}

This study aimed to explore the evolution and spread of DENV-1 genotype I virus in Southeast Asia and surrounding areas, using phylogenetic analysis methods to draw migration maps (Figure 2, Figure 3). Blue, red and Purple indicates that the support rate for this virus migration path in the experimental results has gradually increased. The migration path map shows that the DENV-1 genotype I dengue virus was introduced in Zhejiang, Fujian, Guangdong, Guangxi, and Yunnan in China during 2010-2014. In addition to Zhejiang Province, there have also been cases of virus emigration in the other three provinces. The domestic migration situation was that the virus introduced from Fujian to Guangdong, and Guangdong to Guangxi.

According to the results of the BF factor, Sri Lanka, Japan, Vietnam, Singapore and New Caledonia were the main sources of the virus migration with total of 14 statistically significant dengue virus migration incidents occurred during 2010-2014. During 2015-2019, DENV1 genotype I virus was introduced in Henan and Guangdong in China, and the source of dengue virus was India. Dengue virus introduced from Guangdong to the neighboring Jiangxi Province, Zhejiang Province, and Thailand, Distributed in a spread pattern. Guangdong played an important role in the domestic spread of the virus. India was the main source of foreign virus migration. And there have been 8 cases of virus transmission related to India in the past 5 years. India and Guangdong were the main sources of virus migration abroad and domestic respectively.

Table. 3 The analysis results of Bayesian phylogenetic geography of 2010-2014 virus sequence.(BF $>3$, indicator $>0.5$ ).

\begin{tabular}{ccc}
\hline From & To & Bf \\
\hline Guangdong & Guangxi & 15.73351 \\
Guangxi & Indonesia & 9190.145 \\
Zhejiang & Vietnam & 15.0326 \\
Sri Lanka & Singapore & 576.0903
\end{tabular}

\begin{tabular}{|c|c|c|}
\hline Sri Lanka & Fujian & 56.22807 \\
\hline Japan & India & 35.62205 \\
\hline Fujian & Vietnam & 132.7939 \\
\hline Vietnam & India & 27.59781 \\
\hline Sri Lanka & Guangdong & 9190.145 \\
\hline Fujian & Guangdong & 100.7636 \\
\hline Singapore & Guangxi & 1045.51 \\
\hline New Caledonia & Indonesia & 19.98848 \\
\hline Singapore & Zhejiang & 22.19997 \\
\hline Singapore & Thailand & 16.01075 \\
\hline Japan & Malaysia & 48.2216 \\
\hline New Caledonia & Yunnan & 407.9917 \\
\hline Fujian & Japan & 46.44037 \\
\hline New Caledonia & Fujian & 16.27142 \\
\hline \multicolumn{3}{|c|}{$\begin{array}{l}\text { Table. } 4 \text { The analysis results of Bayesian phylogenetic } \\
\text { geography of } 2015-2019 \text { virus sequence. }(\mathrm{BF}>3 \text {, indicator }>0.5) \text {. }\end{array}$} \\
\hline From & To & $\mathrm{Bf}$ \\
\hline India & Bhutan & 34.0185 \\
\hline India & Guangdong & 52.74934 \\
\hline India & New Caledonia & 63.95552 \\
\hline India & Indonesia & 55304.79 \\
\hline Guangdong & Thailand & 44.44415 \\
\hline New Caledonia & Myanmar & 122.1366 \\
\hline India & Henan & 16.69327 \\
\hline Guangdong & Jiangxi & 13.9191 \\
\hline Australia & India & 54.23554 \\
\hline Thailand & India & 22.24948 \\
\hline Myanmar & India & 31.19695 \\
\hline Myanmar & Australia & 13.23572 \\
\hline Indonesia & Vietnam & 68.05303 \\
\hline Guangdong & Zhejiang & 19.67464 \\
\hline
\end{tabular}

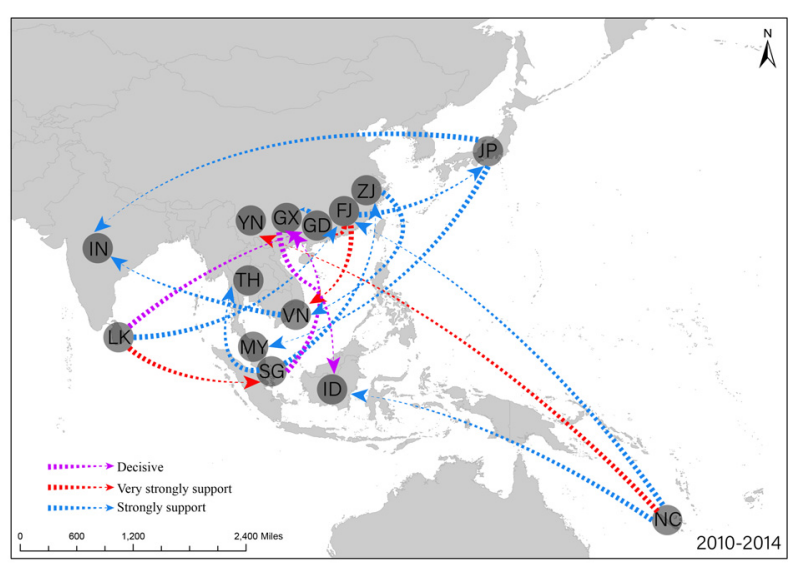

Fig.2 Spatial diffusion of DENV-1 genotype 1 viruses in 20102014.Coloured lines indicated statistically supported migration rates with a mean indicator of $>0.5$. Purple arrows, decisive rates with $\mathrm{BF}>1,000$; Red arrows, very strongly supported rates with $100<\mathrm{BF}<1,000$; Blue arrows, strongly supported rates with $10<\mathrm{BF}<100$ 


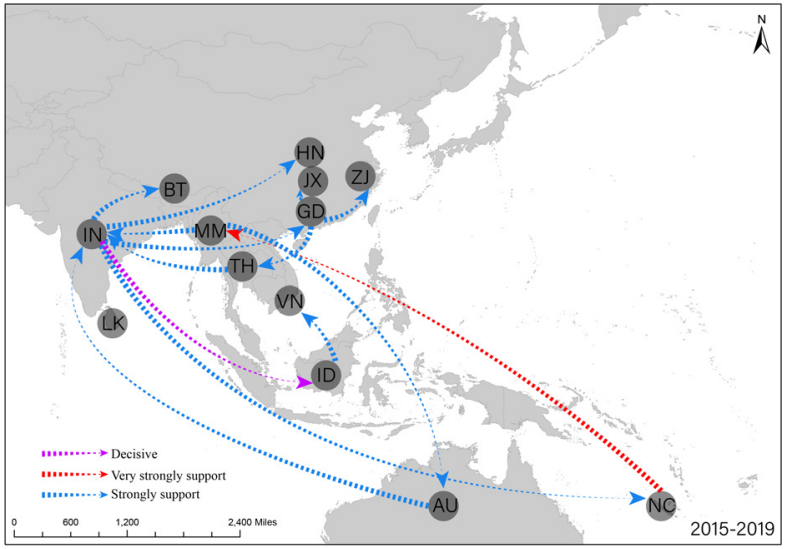

Fig.3 Spatial diffusion of DENV-1 genotype 1 viruses in 20152019.

\section{Discussion}

The disease caused by the dengue virus is widespread in Africa, America, Southeast Asia, and the Western Pacific including China On a global scale. The dengue virus circulating in this area not only covers all serotypes, but each serotype can be subdivided into different genotypes [11]. All four serotypes of dengue virus have been prevalent in coastal provinces in my country, and imported patients are diagnosed almost every year, among which DENV-1 has the highest number of occurrences. The dengue virus outbreak in China is an outbreak and epidemic of local cases caused by imported cases [12]. Imported cases in China mainly come from Southeast Asia (10 consecutive years (2004-2013) accounted for more than $80 \%$ of all imported cases [13]. In 2014,a large-scale dengue fever outbreak in Guangdong Province. DENV-1, DENV2, and DENV-3 were infected with multiple types, mainly DENV-1[2].In this study: 2010-2014, the domestic transmission of DENV-1 genotype 1 virus was introduced from Fujian to Guangdong and Guangdong to Guangxi. With a tendency to migrate to the inland.From 2015 to 2019, India played an significant role in the migration of the virus.The dengue migration potential is gradually increasing during the recent years when compared with decades ago .This may be due to the rise of temperatures in India[14]. Climate changing might be a foremost factor for increasing the risk of dengue in Indian [15].

This study analyzed the $\mathrm{E}$ gene sequence data of DENV-1 genotype I virus in the Asia-Pacific region from 2010 to 2014 and 2015 to 2019 , and constructed the dynamic transmission process of the genotype virus during these two periods. The transmission mode, evolutionary pattern and general distribution of the dengue virus of this genotype provide a certain reference basis for the prevention and control of dengue disease.

\section{References}

1. S.Chen The origin of dengue viruses caused the DF outbreak in Guangdong province, China, in 2006.J.
Infection Genetics \& Evolution, 11,5:11831187(2011)

2. H.B.Chen,J.Z.Wang,et al.Sequence analysis of e genes of dengue viruse isolated from Fujian in 20122014.J.Chinese Preventive Medicine , 17 ,6 (2016)

3. S.Bhatt, P.Gething,O.Brady,et al. The global distribution and burden of dengue.J. Nature 496, 504-507 (2013)

4. WHO Fact Sheet. Dengue and Severe Dengue. WHO.(2018)

5. O.M.Kolawole,A.A.Seriki,A.A.Irekeola, K.E.Bello, O.O.Adeyemi. Dengue virus and malaria concurrent infection among febrile subjects within Ilorin metroplis,Nigeria.J.Med. Virol. 89, 1347-1353. (2017)

6. P.J.Bujalowski, W.Bujalowski,K.H.Choi.Interaction $\mathrm{s}$ between the dengue virus NS5 and stem-loop A. J. Virol. 91, 1-11.(2017)

7. A.J.Drummond, A.Rambaut. BEAST: Bayesian evolutionary analysis by sampling trees.J.BMC Evol. Biol. 7, 214. (2007)

8. K.Katoh, G.Asimenos, H.Toh. Multiple Alignment of DNA Sequences with MAFFT. In: Posada D. (eds) Bioinformatics for DNA Sequence Analysis.J. Methods in Molecular Biology (Methods and Protocols), vol 537. (2009)

9. P.Lemey,A.Rambaut, A.J.Drummond, M.A.Suchard. Bayesian phylogeography finds its roots.J.PLoS Comput Biol.59.(2009)

10. Y.C.F.Su, J.Bahl, U.Joseph, et al. Phylodynamics of H1N1/2009 influenza reveals the transition from host adaptation to immune-driven selection.J. Nature Communications, 6:7952(2015)

11. A.P.Goncalvez,A.A. Escalante ,F.H. Pujol, et al. Diversity and Evolution of the Envelope Gene of Dengue Virus Type 1.J. Virology, 303(1):110119(2002)

12. Q.Y.Liu.Dengue fever in China: new epidemical trend, challenges and strategies for prevention and control.J. Chinese Journal of Vector Biology and Control. $31,1(2020)$

13. W.Y.Ning, et al .Spatial and Temporal Variations of Dengue Fever Epidemics in China from 2004 to 2013.J. Journal of GeoInformation Science.017.005.(2015)

14. S.G. Kakarla , K.R.Bhimala, M.R.Kadiri, et al. Dengue situation in India: Suitability and transmission potential model for present and projected climate change scenarios.J. ence of The Total Environment, 739:140336(2020)

15. Y.A.Halasa,V.Dogra,N.Arora,et al.Overcoming data limitations:design of a multi component study for estimating the economic burden of dengue in India. J.Dengue Bull 35, 1-14(2011) 